\title{
AN OPTIMIZED METHOD FOR THE DETERMINATION OF VOLATILE AND SEMI-VOLATILE ALDEHYDES AND KETONES IN AMBIENT PARTICULATE MATTER
}

\author{
JOHN LIGGIO and ROBERT MCLAREN* \\ Centre for Atmospheric Chemistry and Chemistry Department, York University, \\ 4700 Keele St, Toronto, Ontario, M3J 1P3, Canada
}

(Received 12 November 2002; In final form 11 June 2003)

\begin{abstract}
A method has been developed to measure aldehydes and ketones associated with atmospheric particles. Carbonyl compounds from particulate material collected on Teflon-coated glass-fiber filters were simultaneously extracted and derivatized with an appropriate 2,4-dinitrophenylhydrazine (2,4-DNPH) solution. neously extracted and derivatized with a various $2,4-\mathrm{DNPH}$ concentrations and solvent compositions was The 13 carbonyl compounds of atmospheric importance. These include formaldehyde, acetaldehyde, studiene, dicarbonyls such as glyoxal and methylglyoxal, and biogenic carbonyls such as pinonaldehyde and acetone, dicarbonyls such as glyoxal and methylglyoxal, and biogenic carbonyls such as pinten $140 \%$ water, and nestones (83-100\% recovery). pH 3 was most efficient in extracting and derivatizing thethods were developed that afforded detection Improved sample enrichment and 2,4-DNPH purification methods were developed that afforded devere $1.9-10.1 \%$. Carbonyl compounds in ambient particulate samples were quantified during a recent field study. Median values for nine carbonyl species ranged from $0.01-33.9 \mathrm{ng} \mathrm{m}^{-3}$ during the study.
\end{abstract}

Keywords: Carbonyls; Dinitrophenylhydrazine; Atmospheric aerosols; HPLC

\section{INTRODUCTION}

The focus of atmospheric chemistry is shifting toward the study of secondary organic aerosol (SOA) formation via gas-particle partitioning processes. These processes are increasingly important in light of the fact that particulate material can play a role in areas such as climate change [1-3] and visibility [4-6]. Several studies have demonstrated that increased particle levels can also cause adverse health effects [7-9], which is particularly relevant for particles containing compounds derived from the photo-oxidation of organic species. These compounds primarily partition into smaller particles, which are able to penetrate the respiratory system. Much of the study with respect to secondary organic species in aerosols focuses on low-volatility products, which are expected to easily partition into the particle phase. This expectation is rooted in existing

*Corresponding author. Fax: +1-416-7365936. E-mail: rmclaren@yorku.ca

ISSN 0306-7319 print: ISSN 1029-0397 online (C) 2003 Taylor \& Francis Ltd DOI: $10.1080 / 03067310310001597653$ 
gas-particle partitioning theory, which is largely based on the vapor pressure of the species in question [10-13]. However, deviations from partitioning theory are now being reported for volatile and semi-volatile products of toluene [14] and $\alpha$-pinene [15] oxidation.

In particular, aldehydes and ketones can contribute significantly to this deviation $[14,15]$. Recent work has also suggested that the additional partitioning of carbonyls to particulate material may occur via a chemical transformation of the parent carbonyl to low-volatility products in the aerosol [16]. Possible mechanisms for this include hydration, polymerization and acetal/hemiacetal formation, which can be catalyzed in the presence of acid [16]. Heterogeneous reactions of this type, with carbonyls, imply that SOA yields may be significantly larger than those predicted by current partitioning theory, which is especially relevant given that many organic photooxidation products contain carbonyl functionality. With this in mind, there are surprisingly few measurements of carbonyl-containing species in ambient particulate material, and even fewer methods available that are geared specifically to this functional group. Existing ambient measurements are usually limited to dicarbonyls such as glyoxal [17,18], methylglyoxal [17,18] and pinonaldehyde [19-22] or to aerosols produced during laboratory studies [14,15,23-25]. Detection of carbonyls in particulate material typically utilizes methods that involve several derivatizing agents, resulting in the conversion of many of the functional groups present [17-19,23]. The simultaneous derivatization of carbonyl, carboxyl and hydroxyl functional groups can significantly increase the complexity of the resultant chromatograms. Utilizing a more selective derivatizing agent can aid in targeting carbonyl-containing species only. The most common derivatizing agent for aldehydes and ketones is 2,4-dinitrophenylhydrazine $(2,4-\mathrm{DNPH})$. It has been used extensively for the determination of gas-phase carbonyl species [26-30], but only rarely for the measurement of aldehydes and ketones associated with aerosols [31-33].

A method utilizing 2,4-DNPH to derivatize particulate-phase carbonyl species is presented here, including all aspects relevant for routine analysis. A description of the process whereby particulate samples collected on filter material are extracted, derivatized with a 2,4-DNPH solution, and analyzed by HPLC/UV absorption is presented. The anticipated advantage of this simultaneous approach over conventional post-extraction derivatization methods is that the derivatization reaction likely facilitates the extraction of carbonyls from particulate material. Optimal conditions for this extraction/derivatization, the efficiency thereof, and the analytical uncertainties associated with several aspects of the method are also discussed. Significant advances have been made in the areas of pre-concentration, extraction, and reagent purification, which afford improved detection limits over past methods and permitted the method to be used during a recent field study in the Lower Fraser Valley, B.C., Canada. Several particulate-phase carbonyl compounds are successfully quantified, and preliminary data from this field campaign are presented and discussed.

\section{EXPERIMENTAL}

\section{Liquid Chromatography and Sampling}

Particulate samples were collected using a conventional high-volume sampler at approximately $1 \mathrm{~m}^{3} \mathrm{~min}^{-1}$ for periods ranging from 8 to $24 \mathrm{~h}$. Teflon-coated 
glass-fiber filters $\left(8^{\prime \prime} \times 10^{\prime \prime}\right.$, Pall-Gellman, Pallflex Fiberfilm) were used for all laboratory and field experiments. Sampled filters were cut into eight equal portions, and unused portions were sealed in aluminum foil and frozen until analysis. Prior to sampling, filters were pre-cleaned by solvent washing with dichloromethane, followed by heating at $290^{\circ} \mathrm{C}$ under a constant flow of UHP helium $\left(10-15 \mathrm{~mL} \mathrm{~min}^{-1}\right)$. Clean filters were stored in self-made Teflon bags, which were heat sealed, and only opened when loading the filter onto the sampler.

Extracts were injected onto an HP 1100 HPLC, equipped with a Rheodyne 7125 injector and a variable wavelength UV absorption detector (Hewlett Packard). Detection wavelengths of $360-440 \mathrm{~nm}$ were used, depending upon the hydrazone of interest and the experiment performed. Separation of the hydrazones was achieved with a Supelcosil LC-18 micro-column $(25 \mathrm{~cm} \times 2.1 \mathrm{~mm}, 3 \mu \mathrm{m}$, Supelco $)$. A solvent gradient program was used: $60 \% \mathrm{~A} / 40 \% \mathrm{~B}$, to $50 \% \mathrm{~A} / 50 \% \mathrm{~B}$ in $42 \mathrm{~min}$, to $100 \% \mathrm{~B}$ in $72 \mathrm{~min}$, where $\mathrm{A}=\mathrm{H}_{2} \mathrm{O}$ and $\mathrm{B}=$ acetonitrile. Hydrazones derived from ambient samples were identified by their respective retention times, and by their absorbance spectra. Where necessary, spectra were obtained by injecting the sample repeatedly at 10-15 different wavelengths.

All carbonyls utilized were purchased from commercial sources (Sigma-Aldrich, Lancaster) at the highest purity available, with the exception of pinonaldehyde. Pinonaldehyde was synthesized via an oxidative bond cleavage of pinandiol (Sigma) described elsewhere [34]. HPLC grade acetonitrile (Sigma) and water (18.2 M $\Omega$, $1 \mathrm{ppb}$ TOC, Milli-Q) were used as solvents. 2,4-dinitrophenylhydrazine (Sigma) was recrystallized three times in acetonitrile, dried under vacuum for $2-3 \mathrm{~min}$ and in an oven for 5-6 min at $60^{\circ}$ C. 2,4-dinitrophenylhydrazones were synthesized as described elsewhere [35]. Standard hydrazone solutions for calibration were prepared by dissolving the appropriate mass of hydrazone in a known volume of acetonitrile. Calibration curves were constructed by plotting the peak area at $390 \mathrm{~nm}$ against the moles of hydrazone injected. Regression analysis was performed $\left(R^{2}>0.99\right)$ to obtain response factors.

Although the extracted carbonyls can be directly injected onto the HPLC system, few peaks other than formaldehyde and acetaldehyde can be observed without some form of pre-concentration. Consequently, an approach was used that permitted the use of minimal $\mathrm{H}_{2} \mathrm{O}$ in the extraction solution, while maintaining an adequate pre-concentration. A guard column (Supelco, $2 \mathrm{~cm} \times 2.1 \mathrm{~mm}, 5 \mu \mathrm{m}$ LC-18) within a guard column holder or cartridge (Supelco) was inserted as a sample loop in an HPLC injector (Fig. 1). The extracted and derivatized carbonyls were filtered, then injected directly onto this guard column, where the hydrazones were trapped. The entire contents of the cartridge were then flushed in the reverse direction, onto the analytical column, where separation of the hydrazones occurred.

\section{Extraction and Derivatization Methods}

Particulate-phase carbonyls were simultaneously extracted and derivatized using 2,4-dinitrophenylhydrazine (2,4-DNPH) in an acetonitrile $/ \mathrm{H}_{2} \mathrm{O}$ solvent mixture. Three approaches were explored with respect to this process. In the first approach (Method 1), one-eighth of an entire filter was placed in a round-bottom flask, together with $25 \mathrm{~mL}$ of a $1 \times 10^{-3} \mathrm{M}$ solution of 2,4-DNPH in $65 \% \mathrm{H}_{2} \mathrm{O}$ and $35 \%$ acetonitrile, 


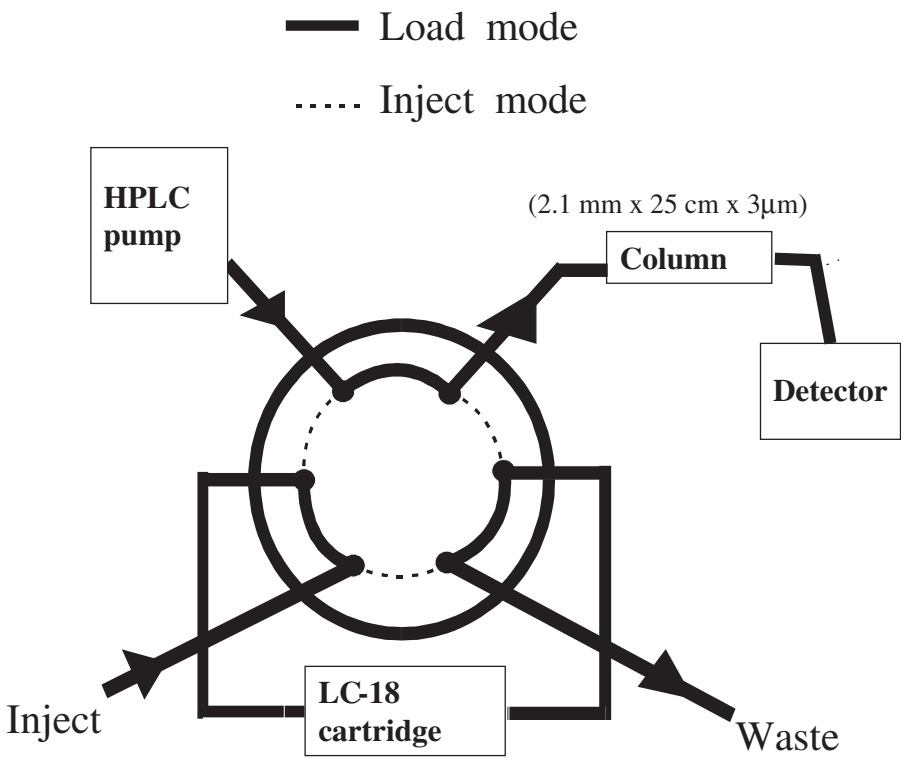

FIGURE 1 Schematic of pre-concentration method. In the load mode, extract is injected onto the C-18 cartridge, where hydrazones are trapped and solvent goes to waste. The valve is rotated to the inject mode, and the entire contents of the cartridge is flushed onto the analytical column followed by separation and detection.

and a $\mathrm{pH}$ of 3 . This extract was refluxed to approximately $80^{\circ} \mathrm{C}$ under a constant stream of UHP helium for $24 \mathrm{~h}$. Extracts were then filtered using small volume syringe filters with a PTFE membrane (Supelco, $13 \mathrm{~mm}, 0.2 \mu \mathrm{m}$ or $4 \mathrm{~mm} 0.5 \mu \mathrm{m}$ ), at which point it was directly injected onto the HPLC. Experiments performed on these syringe filters indicated that no hydrazones were lost during this filtering process.

Identical conditions were utilized in the second approach (Method 2) except that the processes were performed in a sealed vessel. One-eighth of a filter and $25 \mathrm{~mL} 2,4-\mathrm{DNPH}$ solution $\left(1 \times 10^{-3} \mathrm{M}\right.$ DNPH, $65 \% \mathrm{H}_{2} \mathrm{O}, 35 \% \mathrm{ACN}$, pH 3$)$ was sealed in an amber glass vessel with a phenolic cap and Teflon liner (Supleco), to avoid any evaporative losses of the carbonyl species. The derivatization was performed at a slightly lower temperature than in Method $1\left(50^{\circ} \mathrm{C}\right)$, with a standard heat block (VWR) to avoid over pressurizing the vessel.

Finally, a third approach (Method 3), performed under sealed conditions but with a significantly greater 2,4-DNPH concentration and smaller $\mathrm{H}_{2} \mathrm{O}$ solvent fraction, was explored. In this case, a $3 \times 10^{-2} \mathrm{M} 2,4-\mathrm{DNPH}$ solution in $60 \% \mathrm{ACN}$ and $40 \%$ $\mathrm{H}_{2} \mathrm{O}$, pH 3, was used. The amount of 2,4-DNPH that can be used is limited by its solubility in this solvent mixture, but the amount used in this instance yielded a nearly saturated solution. Saturated solutions of 2,4-DNPH in water and acetonitrile are approximately $10^{-4}$ and $10^{-2} \mathrm{M}$ respectively. A magnetic stirring bar was placed in the glass vessel during extraction of ambient samples. Stirring for several minutes quickly broke down the filter material, which may have aided in the mass transfer of carbonyls from particles, and ensured that the extraction solution completely covered the sample. 


\section{2,4-DNPH Reagent Preparation}

A cleaning and storage method was developed specifically for those 2,4-DNPH solutions utilized in this article. However, it can be adapted to other 2,4-DNPH applications. A clean reagent is critical, since most methods involving 2,4-DNPH are primarily blank limited (refer to discussion section). A schematic representation of this system is shown in Fig. 2. The 2,4-DNPH derivatizing solution utilized above $\left(3 \times 10^{-2} \mathrm{M}, 60 \% \mathrm{ACN}, 40 \% \mathrm{H}_{2} \mathrm{O}, \mathrm{pH} 3\right)$ was stored in a custom-made $1-\mathrm{L}$ stainless-steel cylinder, under a constant UHP helium head pressure of 20-30 psi which maintained the cleanliness of the solution. A supply of HPLC grade acetonitrile was also stored in a similar canister under helium pressure. A series of gas toggle valves controlled helium flow, and Tefzel liquid shutoff valves (Supelco) controlled liquid flow. All containers and valves were connected with $1 / 8^{\prime \prime}$ stainless-steel tubing. A $30-\mathrm{cm}$ length of $1 / 4^{\prime \prime}$ stainless-steel tubing was packed with approximately $2 \mathrm{~g}$ of a C-18-coated silica packing material (Supelco, ENVI-18, 17\% C, end-capped) and connected to the various valves. A stainless-steel screen with $10-\mu \mathrm{m}$ openings (Supelco), was cut and placed at the ends of this tubing to prevent the loss of packing material. When a 2,4-DNPH solution was required, helium pressure forced the pre-made solution through this C-18 material, thus removing hydrazone impurities. These impurities were removed because the C-18 material essentially behaves like a

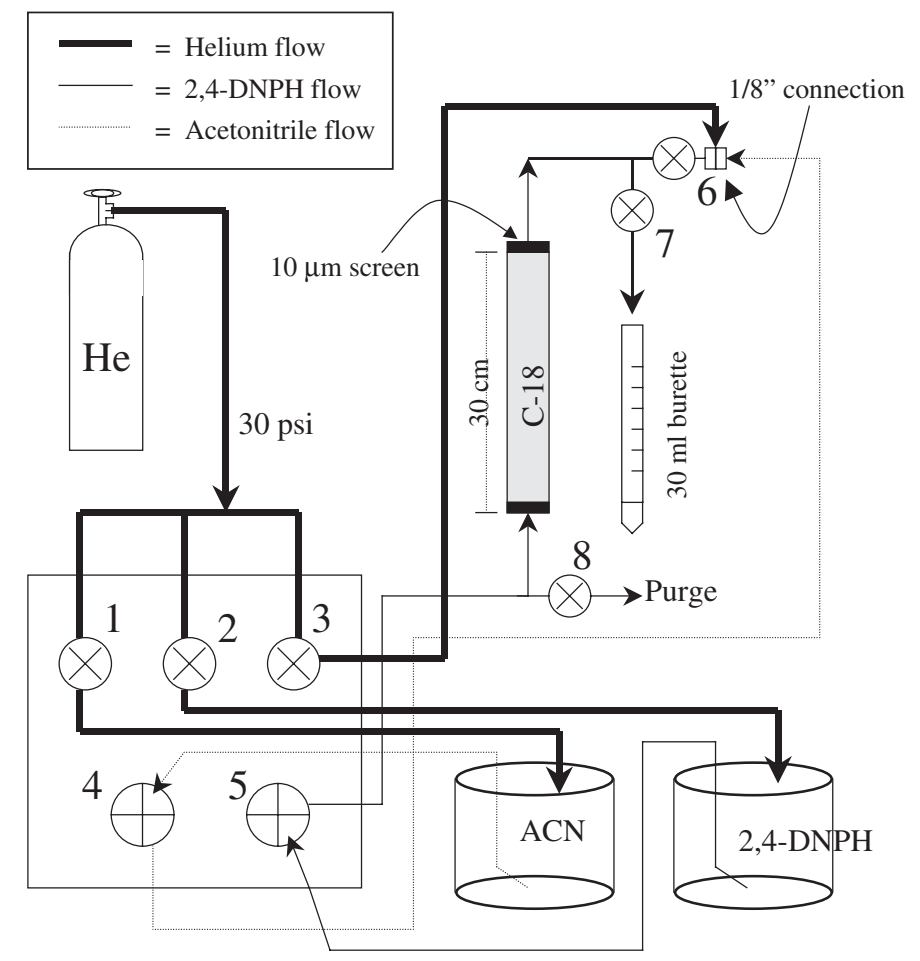

FIGURE 2 Schematic representation of 2,4-DNPH preparation system. Toggle valves 1-3 control He flow to solvent and extraction solution reservoirs. Valves 4,5 control flow of 2,4-DNPH solution, and acetonitrile through C-18 material. Valves 6,7 allow flow to be stopped during regeneration of C-18 with acetonitrile, and drying with He. Valve 8 allows for purging of the system. 
chromatographic column, where 2,4-DNPH is eluted first and all other hydrazones of interest elute after 2,4-DNPH. 2,4-DNPH itself was also slightly retained on this material and, consequently, sufficient amounts of the 2,4-DNPH solution must be forced through the $\mathrm{C}-18$ material to maintain a high concentration of the derivatizing agent. Passing $20 \mathrm{~mL}$ of the 2,4-DNPH solution through the packed column resulted in a solution whose concentration was greater than $90 \%$ of that stored in the stainless steel vessel. The cleaned 2,4-DNPH solution was collected in a $50-\mathrm{mL}$ graduated burette, at which point aliquots of varying amounts were dispensed to the extraction vessels. The C-18 material was regenerated after each delivery by pressurizing the stored acetonitrile through the packed tubing in the reverse direction and out through a purge valve. Approximately $25-30 \mathrm{~mL}$ of acetonitrile was necessary for this process. The packing was then dried with a pressurized helium stream for 3-5 min before another batch was cleaned.

\section{RESULTS AND DISCUSSION}

\section{Extraction and Derivatization Efficiency}

The extraction and derivatization methods outlined in the experimental section were tested to determine their respective efficiencies. Results from these experiments are shown in Fig. 3. In such experiments, one-eighth of a filter (Teflon-coated glass fiber, $8^{\prime \prime} \times 10^{\prime \prime}$ ) was spiked with a carbonyl solution of known concentration at the nanogram level, and extracted using Methods 1-3. Extracts were then analyzed to determine the fraction of the original carbonyl mass that can be recovered from the extraction and derivatization process. It is expected that extraction of a liquid carbonyl from the Teflon filter material does not take a significant amount of time, and therefore the extraction experiments above are more likely a measure of the derivatization efficiency of the particular 2,4-DNPH solution. When utilizing an open vessel (Method 1, Fig. 3A), the derivatization efficiency for aldehydes is typically greater than $80 \%$. However, ketones, (acetone, acetophenone) or dicarbonyls with a ketone functional group (methylglyoxal and biacetyl), exhibit a very low derivatization efficiency $(5-50 \%)$. Acetaldehyde also exhibits this low efficiency. There are several explanations for these low recoveries/efficiencies. Although the extraction system is heated under reflux in Method 1, it may be possible that the some of the more volatile carbonyls are flushed away by the constant flow of helium. This possibility is the impetus for exploring Method 2, which utilizes sealed conditions for the extraction.

Although when using Method 2 the derivatization efficiency for ketones is slightly increased, they remain rather low (Fig. 3B). However, the acetaldehyde efficiency increases dramatically to $96 \% \pm 8 \%$, indicating that volatilization is largely responsible for the low acetaldehyde efficiency observed in Method 1. This tends to agree with its physical properties, since acetaldehyde has the lowest Henry's law constant of those carbonyls studied and is highly volatile. A typical effective Henry's Law constant for acetaldehyde can range from $11-17 \mathrm{M} \mathrm{atm}^{-1}$ [36]. Of the remaining carbonyls studied here, acetone has the Henry's law constant most similar to acetaldehyde $\left(22-35 \mathrm{M} \mathrm{atm}^{-1}\right)$ [36], while compounds such as formaldehyde $\left(3 \times 10^{3}-7 \times\right.$ $\left.10^{3} \mathrm{M} \mathrm{atm}^{-1}\right)$ [36] and glyoxal $\left(3.6 \times 10^{5} \mathrm{M} \mathrm{atm}^{-1}\right)$ [36] have Henry's law constants that are orders of magnitude greater. 

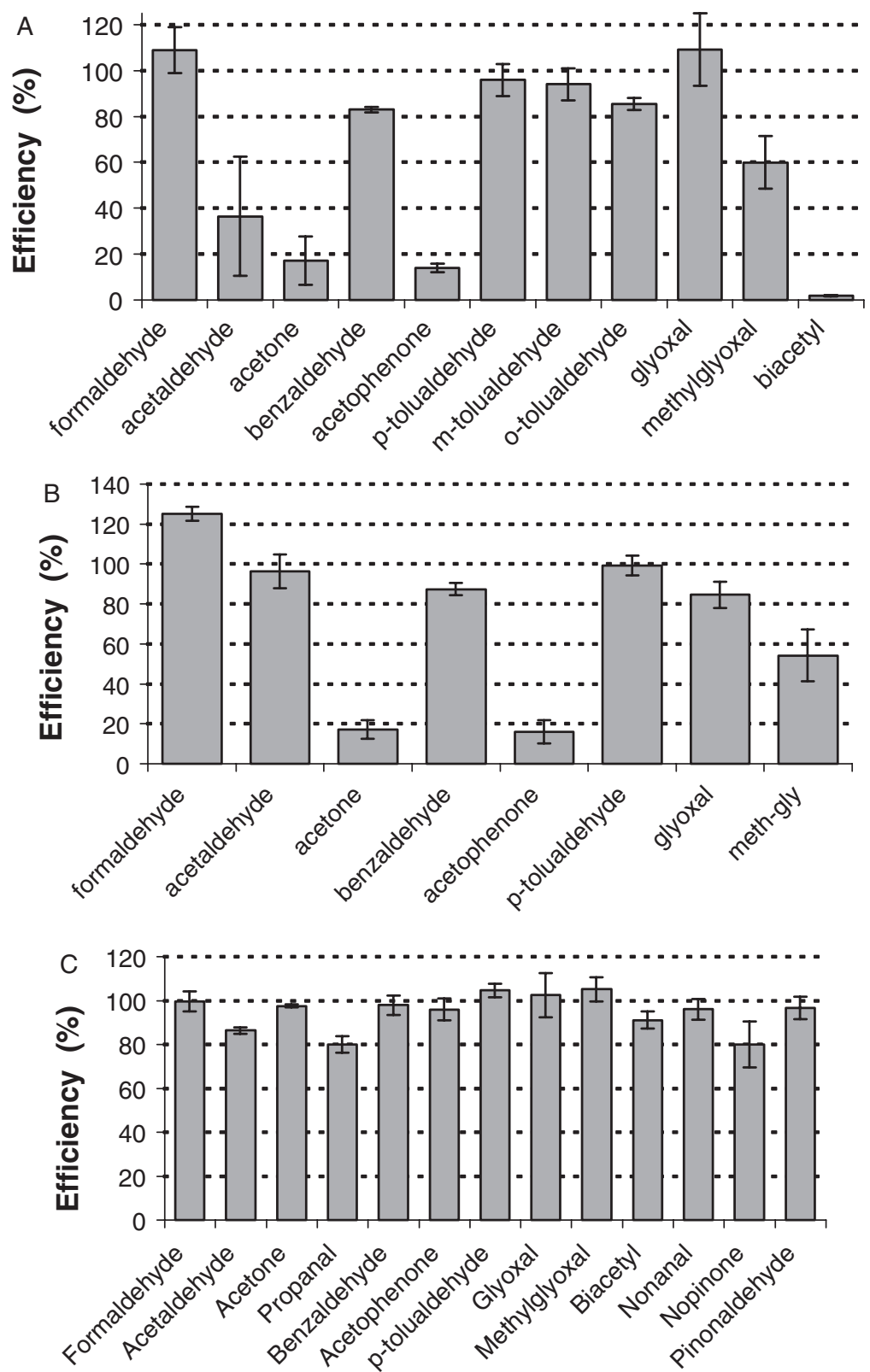

FIGURE 3 Extraction/derivatization efficiency for selected carbonyl compounds. A, Using method 1; $\mathrm{B}$, using method 2; C, using method 3 - the final optimized extraction solution. Error bars represent the standard deviation of 3-5 experiments under the same conditions. Method 3 was used during subsequent field studies.

The continued low efficiency for ketones can be rationalized by examining the derivatization reaction itself. The entire system is known to be in equilibrium (Fig. 4). With a large excess of 2,4-DNPH in a pure organic solvent, the equilibrium lies far to the right, in favor of the product hydrazones. However, a very large aqueous fraction 


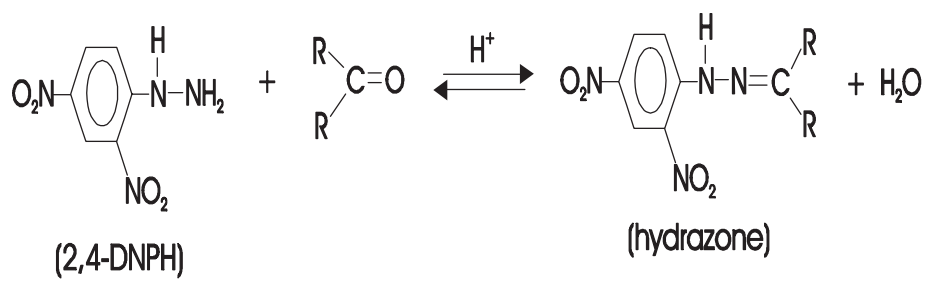

FIGURE 4 Hydrazone derivative in equilibrium with 2,4-dinitrophenylhydrazine (2,4-DNPH).

in the solvent would likely shift this equilibrium back towards the starting materials [33], owing to the reverse reaction, as well as to the decreased solubility of the hydrazones compared to that of 2,4-DNPH. Consequently, with a solvent consisting of $65 \% \mathrm{H}_{2} \mathrm{O}$, it is likely that the reaction is not complete, resulting in the low efficiencies observed. This is especially evident for ketones, whose 2,4-DNPH reaction kinetics are slower than those for aldehydes. Equilibrium constants for the ketone/2,4-DNPH system in an $\mathrm{ACN} / \mathrm{H}_{2} \mathrm{O}$ solvent mixture are also likely small, although no information is currently available. A reduced water fraction, as well as an increased 2,4-DNPH concentration should shift the equilibrium towards products. This was attempted in Method 3, with results shown in Fig. 3C. Under the conditions of Method 3, the efficiencies for all compounds of interest are greatly enhanced and many are close to $100 \%$ within the uncertainties.

The method of pre-concentration, which is discussed below, requires the use of an aqueous solvent in the extraction process. Increasing the amount of water in the extraction solution increases the degree of pre-concentration possible in the post-extraction analysis, but at the same time decreases the solubility of 2,4-DNPH and of the hydrazones formed during the derivatization process. A proper balance of water and acetonitrile is essential to the success of the entire analysis. The 2,4-DNPH concentration $\left(3 \times 10^{-2} \mathrm{M}\right)$ and aqueous solvent fraction $(40 \%)$ used in Method 3 are optimal for the extraction, pre-concentration, and reagent preparation portions of the method, and are used in all subsequent experiments and field studies. Although large aqueous fractions are adequate for the derivatization of aldehydes, for ketone analysis careful consideration of the DNPH and aqueous content is needed.

\section{Length of Extraction}

Derivatization efficiency experiments alone do not completely describe the extraction process, as it is unlikely that carbonyls are extracted from particles collected on filters as easily as they are from spiked filters. The results of an experiment performed to determine the time necessary for a complete extraction from ambient particles are shown in Fig. 5. In this experiment, aerosol samples collected on Teflon-coated glass-fiber filters from a recent field study (see below) are extracted successively using Method $3\left(\mathrm{Temp}=50^{\circ} \mathrm{C}\right)$. The analysis is scaled down to maximize the number of samples available. Filter surface area and extraction volume are equally reduced by a factor of ten, resulting in a filter surface area of $5.29 \mathrm{~cm}^{2}$ and an extraction volume of $500 \mu \mathrm{L}$. Extractions are carried out as outlined for Method 3 above and injected directly onto the HPLC system. A detection wavelength of $390 \mathrm{~nm}$ was used for all injections with the solvent gradient program outlined above. After analysis of several extracts from these extraction-time experiments spanning many hours, it is evident that the extraction 

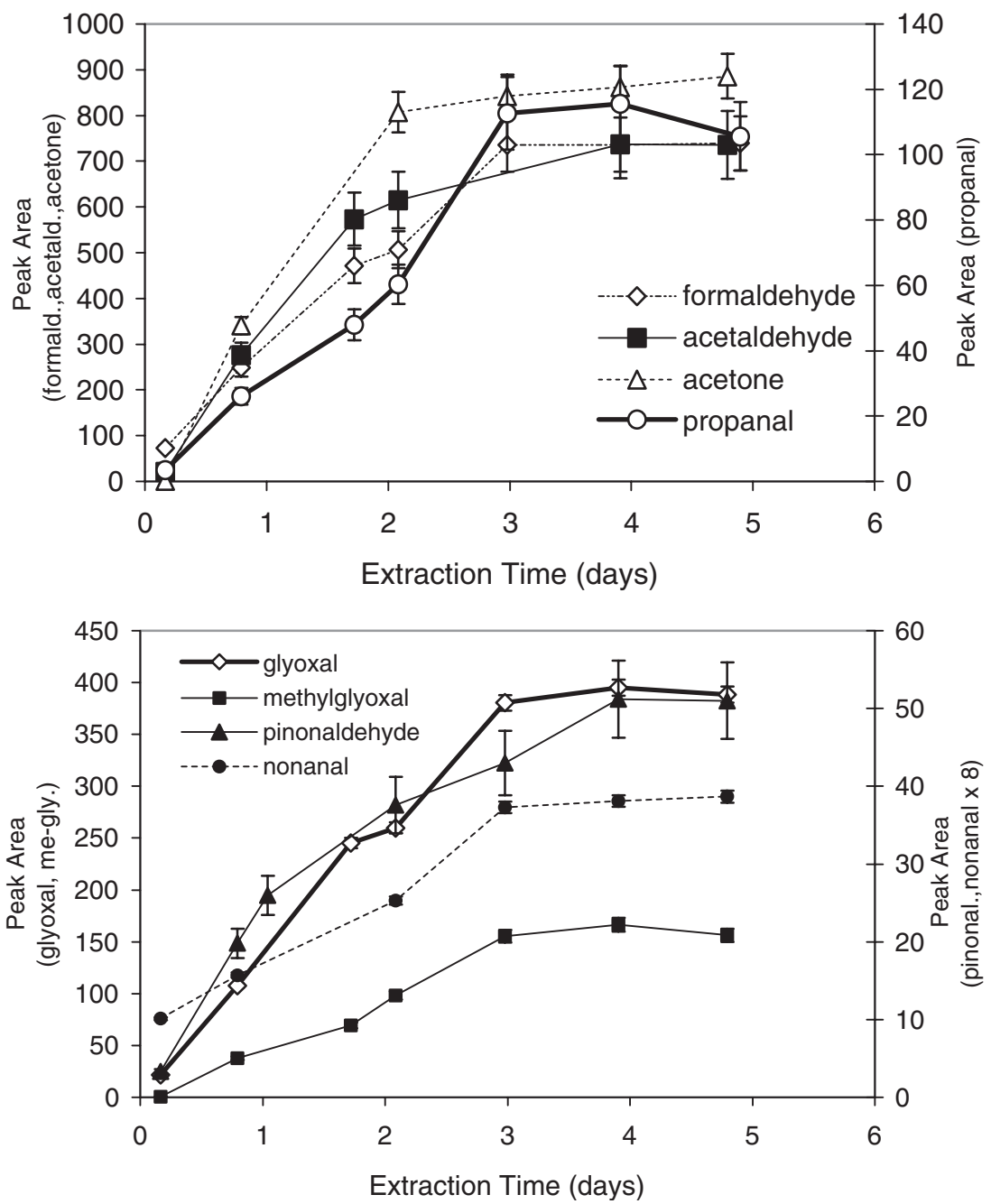

FIGURE 5 Extraction time necessary for various carbonyl compounds. Each point represents the observed signal (peak area) from an extraction and derivatization of an equivalent portion of filter material. Sample was collected in the Lower Fraser valley, B.C. Canada. Error bars represent the relative standard deviation of the entire method, determined previously. Connecting lines are for clarity of presentation only.

of carbonyls from particulate matter in this manner is a slow process, as the measured peak areas for all carbonyls steadily increase over time. Consequently, the experiment was extended until such time that the measured signal became reasonably constant. Analyses of blank 2,4-DNPH solutions alone over the same period of time and under the same conditions show no increase in blank levels, indicating that a slow leak into the vessel does not contribute to the observed signals and that complete extraction is only achieved after several days. This may suggest that the extraction of carbonyls from particulate material is kinetically limited by mass transfer from the center of particles to the surface. It may also be indicative of a slow thermal decomposition of some as yet unidentified polymer, yielding carbonyl monomer units, which has 
recently been hypothesized [16]. Such polymers are thought to arise via the hydration of aldehydes as well as by acetal/hemi-acetal formation in slightly acidified media [16]. The detection of individual carbonyl species may result from the reverse of the above reactions, facilitated by the extraction and derivatization procedures. Artifact formation also cannot be ruled out as a possible explanation, perhaps from some acid-catalyzed conversion or oxidation of other compounds present in the aerosol sample. However, it is likely that such a process requires significant heating, presumably greater than the $50^{\circ} \mathrm{C}$ used in these experiments. A very high temperature for an extended period of time cannot be used for extraction, as 2,4-DNPH decomposes at higher temperatures and pressure. Positive artifact formation from gas-phase adsorption of carbonyls to the filter material is unlikely to account for the long extraction times because carbonyls spiked on filters are found to be completely derivatized, typically in less than $30 \mathrm{~min}$, depending on the species of interest.

\section{Pre-Concentration}

Results from experiments performed using the pre-concentration method described above (refer to experimental section), to determine the maximum volume that can be injected without significant peak broadening while achieving a significant enrichment, are shown in Fig. 6. This was accomplished by injecting standard hydrazone solutions containing 40,50, and $60 \% \mathrm{H}_{2} \mathrm{O}$ onto the guard cartridge, with a solvent gradient program of: $60 \% \mathrm{~A} / 40 \% \mathrm{~B}$ to $100 \% \mathrm{~B}$ in $30 \mathrm{~min}$, where $\mathrm{A}=$ water and $\mathrm{B}=$ acetonitrile. A flow rate of $0.2 \mathrm{~mL} \mathrm{~min}^{-1}$ was used, and hydrazones were detected at $390 \mathrm{~nm}$. Standard hydrazone concentrations for each run are adjusted so as to ensure that the number of moles of hydrazone injected remained the same regardless of the injection volume. Approximately $1 \times 10^{-10} \mathrm{~mol}$ of hydrazone is injected. Although water reduces the efficiency of derivatization, it also increases the ability of the guard cartridge to trap hydrazones in a narrow band, thereby decreasing peak widths on the chromatographic column. This quality is evident in Fig. 6, where peak widths tend to broaden with decreasing $\mathrm{H}_{2} \mathrm{O}$ content, a less advantageous situation from a purely chromatographic standpoint. However, peak broadening at the lowest $\mathrm{H}_{2} \mathrm{O}$ fraction shown here $(40 \%$, Fig. 6C) is not so severe that an adequate pre-concentration cannot be achieved. A $40 \% \mathrm{H}_{2} \mathrm{O}$ extraction solution continues to permit injections of more than $100 \mu \mathrm{L}$ without significant peak broadening (Fig. 6C), while being advantageous for the derivatization process. Low molecular weight carbonyls such as acetaldehyde require a smaller injection volume at the $40 \% \mathrm{H}_{2} \mathrm{O}$ level to maintain narrow peak widths, owing to the slightly more polar nature of their corresponding hydrazones. Hydrazones of dicarbonyls such as glyoxal, are quite non-polar, and therefore are retained on the LC-18 guard column more efficiently than other hydrazones. As a result, peak widths for glyoxal-DNPH do not increase significantly even with $200-\mu \mathrm{L}$ injections at $40 \% \mathrm{H}_{2} \mathrm{O}$. An injection of $40-50 \mu \mathrm{L}$ is sufficient to concentrate both low and high molecular weight carbonyl-DNPH compounds at an acceptable level. Typically, the use of a 2.1-mm i.d. column with a standard sample loop, is limited to injection volumes of no more than $5 \mu \mathrm{L}$. Injecting $50 \mu \mathrm{L}$ in the manner suggested here, maintains chromatographic resolution and can easily enrich the sample by an order of magnitude $(10 \times)$, significantly more than conventional $5 \mu \mathrm{L}$ injections. If aldehydes alone were to be analyzed, then a larger aqueous content could be used for extraction and derivatization, resulting in even larger pre-concentration ratios 

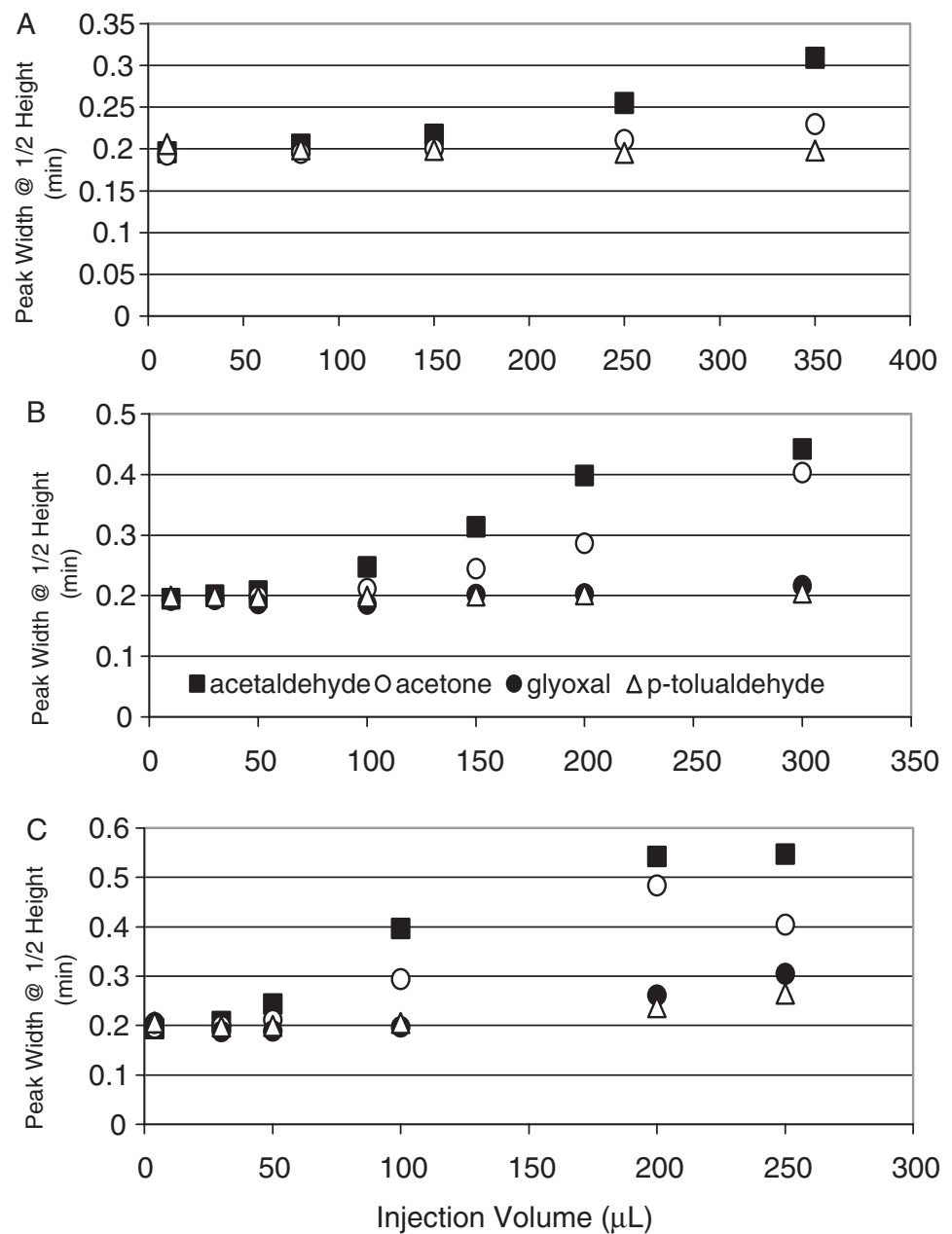

FIGURE 6 Effect of increasing injection volumes on the peak width at half height for low and high molecular weight hydrazones, at a given water fraction. A, $60 \%$ water, $40 \%$ acetonitrile; $\mathrm{B}, 50 \%$ water, $50 \%$ acetonitrile; $\mathrm{C}, 40 \%$ water, $60 \%$ acetonitrile.

$(40-50 \times)$. To further increase sensitivity, extraction volumes were reduced from 25 to $5 \mathrm{~mL}$. This has no effect on the extraction or derivatization efficiencies, since the number of moles of 2,4-DNPH derivatizing agent remains many orders of magnitude greater than the moles of carbonyls present in the samples.

Several methods are available to enrich extracts. The most common pre-concentration methods involve either a nitrogen blow-down followed by dissolution into a smaller volume of solvent $[17,18,23,24]$, or solid-phase extraction (SPE) with commercially available C-18 coated silica cartridges [31,37-39]. Blow-down of solvent may cause the equilibrium to shift to starting materials and result in volatile carbonyls being blown away. Also, the reduced volume increases the concentration of the acid to extremely high levels. Such acidic media cannot be injected onto octadecyl silica stationary phases, and degradation of derivative products at very low $\mathrm{pH}$ has been 
observed [39]. Solid-phase extraction on available C-18 silica cartridges is a possible alternative. However, a reasonable enrichment with SPE requires that the extract be fairly polar in nature, typically with aqueous fractions greater than $70 \%$ [31,37-39]. Since high aqueous fractions are not conducive to the analysis of ketones by 2,4-DNPH, SPE is not a feasible alternative. The enrichment method described in this paper has the advantage of being able to pre-concentrate analytes sufficiently, without the need of extremely large amounts of water. Also, in the inject mode, the entire contents of the cartridge are flushed onto the analytical column, avoiding an elution step required with SPE. In addition, the smaller packing material $(5 \mu \mathrm{m})$ in guard columns compared to that of SPE tubes $(\sim 50 \mu \mathrm{m})$ results in a greater hydrazone retention while reducing the amount of water necessary in extracts. The degree of pre-concentration is dependent upon the volume injected onto the guard cartridge with a given aqueous content. Increasing this volume will increase the effective preconcentration at the expense of increasing the observed chromatographic peak widths. This aspect is extremely important, as one of the goals of basic chromatography is to maintain narrow peak widths, since broadening of peaks can result in significantly lower sensitivity, higher detection limits, and inadequate separations.

\section{2,4-DNPH Reagent Purification Methods}

The efficiency of the 2,4-DNPH extraction solution purification process was investigated by injecting 2,4-DNPH solutions that were and were not subjected to this procedure. A comparison of the chromatograms for 2,4-DNPH extraction solutions cleaned in the aforementioned manner with those that were not is shown in Fig. 7. Analytical methods involving 2,4-DNPH invariably suffer from blank problems. Over time, solutions of 2,4-DNPH become contaminated, and background levels of hydrazones in these solutions are difficult to remove. A large amount of hydrazone contaminants in the blank increases the uncertainty of any following quantification and compound identification. Background hydrazone levels are even more critical in this method due to the large injection volumes, which pre-concentrate analytes as well as contaminants. Commonly, 2,4-DNPH solutions are freed from hydrazone impurities by successive extractions with $\mathrm{CCl}_{4}[32,40,41]$. However, extraction of hydrazones impurities into a $\mathrm{CCl}_{4}$ layer requires that the 2,4-DNPH solution be prepared in water. Since hydrazones are more soluble in $\mathrm{CCl}_{4}$ than in water, they are effectively removed. DNPH solutions prepared in some fraction of organic solvent are not easily cleaned in this manner, as the organic solvent is usually soluble in the $\mathrm{CCl}_{4}$. Purification of the 2,4-DNPH solution in the manner presented here allows a significant portion of the solution to be organic in nature, while continuing to provide an adequate cleaning.

Results of these experiments indicate that a solution containing $40 \% \mathrm{H}_{2} \mathrm{O}$ removes higher molecular weight hydrazones more efficiently than low molecular weight (higher polarity) hydrazones such as formaldehyde and acetaldehyde-DNPH. However, in the region of the chromatogram where many of the compounds of interest are eluted (Fig. 7A), a significant improvement is observed. More polar hydrazones can effectively be removed by increasing the fraction of water in the extraction solution to $60 \%$ (Fig. 7B). However, in this case the analysis must then be geared solely to aldehydes. 2,4-DNPH solutions stored in this manner are stable for several weeks, as background levels do not increase during this time (Fig. 7B). 

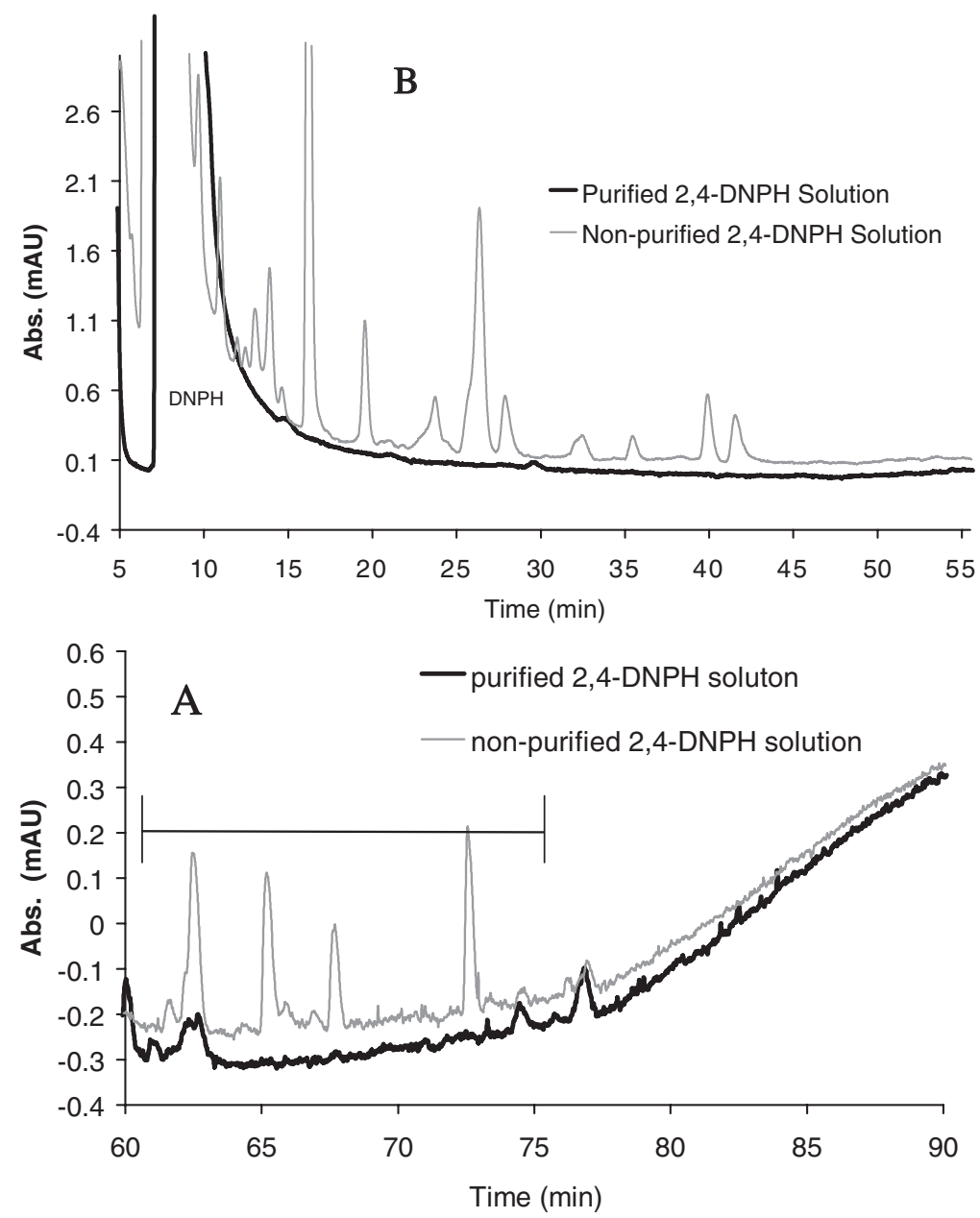

FIGURE 7 Comparison of 2,4-DNPH solution blanks with and without utilizing cleaning method. 40- $\mu \mathrm{L}$ injections, $390 \mathrm{~nm}$ detection, $1 \times 10^{-2} \mathrm{M}$ 2,4-DNPH prepared as above. A, 2,4-DNPH in $60 \%$ acetonitrile, $40 \% \mathrm{H}_{2} \mathrm{O}$ injected with and without cleaning. Bracket indicates area of interest in chromatogram where C5-C10 carbonyls are eluted; $\mathrm{B}, 2,4-\mathrm{DNPH}$ in $60 \% \mathrm{H}_{2} \mathrm{O}, 40 \%$ acetonitrile. Clean blank injection made after two weeks of storage under helium and using the purification method outlined above. Contaminated injection made from same 2,4-DNPH solution left standing in a sealed glass flask for two weeks, and not cleaned as above. Low molecular weight hydrazones are eluted in this region of the chromatogram only.

\section{Reproducibility, Detection Limits, Stability}

Typical detection limits at the $3 \sigma$ level for carbonyls in aerosols during a recent field study are given in Table I. Detection limits for this method are dependent upon the background levels of analytes in cleaned filters and extraction solutions. Although the cleaning and handling procedures used in this study reduced background levels significantly, it remained quite difficult to remove all impurities from the Tefloncoated glass-fiber filters. Although low molecular weight carbonyls such as formaldehyde, acetaldehyde, and acetone cannot be completely removed from the filter or 2,4-DNPH solution, levels of these carbonyls are typically much smaller than those 
TABLE I Detection limits and precision of carbonyl measurement method

\begin{tabular}{lcc}
\hline Carbonyl compound & $\begin{array}{c}\text { Detection } \text { limit }^{\mathrm{a}} \\
\left(\mathrm{ng} \mathrm{m}^{-3}\right)\end{array}$ & $\begin{array}{c}\text { Precision }^{\mathrm{b}} \\
(\%)\end{array}$ \\
\hline Formaldehyde & 5.6 & 7.9 \\
Acetaldehyde & 1.1 & 10.1 \\
Acetone & 0.44 & 5.5 \\
Propanal & 0.27 & 9.9 \\
Benzaldehyde & 0.009 & - \\
Glyoxal & 0.15 & 1.9 \\
Methylglyoxal & 0.010 & 3.8 \\
Biacetyl & 0.009 & - \\
Nopinone & 0.022 & - \\
Pinonaldehyde & 0.023 & 9.7 \\
Nonanal & 0.27 & 2.0 \\
\hline
\end{tabular}

${ }^{\text {a }}$ The detection limits here represent those during the Pacific 2001 field study in the Lower Fraser Valley B.C., Canada. Detection limits are calculated as:

$$
\left(3 \sigma_{\text {blank }} / R\right) \times(\text { Ext. Vol. } / \text { Inj. Vol. }) \times(1 / f) \times(1 / \text { vol. air }) \times \mathrm{MW} \times 10^{9}
$$

$\sigma_{\text {blank }}=$ standard deviation of peak area in blank filter measurements during study $(N=7)$; $R=$ instrument response for hydrazone of interest (peak area/mole injected); Ext. Vol.= extraction volume $(0.005 \mathrm{~L})$; Inj. Vol. = Injection volume $\left(40 \times 10^{-6} \mathrm{~L}\right) ; f=$ fraction of filter used for analysis $(1 / 8)$; vol. air $=$ volume of air sampled $\left(480 \mathrm{~m}^{3}-8 \mathrm{~h}\right.$ at $\left.1 \mathrm{~m}^{3} / \mathrm{min}\right)$; $\mathrm{MW}=$ molecular weight of carbonyl compound $(\mathrm{g} / \mathrm{mol}) ; 10^{9}=$ conversion of grams to nanograms. ${ }^{\mathrm{b}}$ The precision of the method was calculated as the relative standard deviation $(N=4)$ of the peak areas obtained from equivalent portions of a sampled filter from Pacific 2001. RSDs for biacetyl, nopinone and benzaldehyde are absent as they were not detected in the sample.

observed in ambient samples. Since the quality of the filter material and 2,4-DNPH varies from batch to batch, detection limits have also been noted to change slightly. Higher detection limits for lower molecular weight carbonyls illustrates the difficulty in completely removing these compounds from filter material and extraction solutions.

An estimate of the precision of the entire method is obtained by extracting several equivalent pieces of a bulk filter. This estimate of precision includes not only the method precision, but also inhomogeneities of the sample across the surface of the filter. The relative standard deviation as a measure of precision, determined in this manner, ranges from $2-10 \%$ and is shown in Table I. Extracts from ambient samples are stored in a freezer at approximately $-15^{\circ} \mathrm{C}$ after analysis. Analysis of the same extracts one month later reveal that they are stable at this temperature, as concentrations for those carbonyls of interest deviated by less than $10 \%$ over this period. Similarly, particulates collected on a Teflon-coated filter and stored at $-15^{\circ} \mathrm{C}$ for three months show no significant degradation of the carbonyls over this time.

\section{Field Data}

The optimized method was used during the Pacific 2001 field study in the lower Fraser Valley, B.C., Canada during August, 2001. Application of this method proved successful, as most of the carbonyls of interest are above the detection limits stated here and could easily be quantified (Table II). A typical chromatogram obtained during this period is shown in Fig. 8. Particulate samples were collected as outlined above, for $8-12 \mathrm{~h}$ at three sites. Samples from the urban (Vancouver, B.C.), rural (Langley, B.C.) and rural/biogenic (Sumas Mtn.) sites were frozen and analyzed upon return to the laboratory. Although a detailed interpretation of the data has not been made, 
TABLE II Summary of particulate carbonyl measurements from the Pacific 2001

\begin{tabular}{|c|c|c|c|c|c|c|}
\hline \multirow[t]{2}{*}{ Carbonyl compound } & \multicolumn{2}{|c|}{ Vancouver site } & \multicolumn{2}{|c|}{ Langley site } & \multicolumn{2}{|c|}{ Sumas Mtn. site } \\
\hline & Concentration $^{\mathrm{a}}$ & Median & Concentration $^{\mathrm{a}}$ & Median & Concentration $^{\mathrm{a}}$ & Median \\
\hline Formaldehyde & $2.9-41.6$ & 18.0 & $6.8-73.4$ & 33.9 & $5.7-53.2$ & 15.7 \\
\hline Acetaldehyde & - & - & - & - & $0.76-13.2$ & 4.8 \\
\hline Acetone & $0.40-3.50$ & 1.70 & $0.61-7.4$ & 2.3 & $3.9-27.6$ & 12.9 \\
\hline Propanal & $0.19-4.7$ & 1.10 & $0.42-4.8$ & 1.7 & $0.86-5.3$ & 2.3 \\
\hline Glyoxal & $0.47-1.4$ & 0.78 & $0.43-3.0$ & 1.2 & $0.55-3.3$ & 1.7 \\
\hline Methylglyoxal & $0.03-0.26$ & 0.10 & $0.064-0.40$ & 0.14 & $0.15-1.2$ & 0.47 \\
\hline Nopinone & $0.002-0.03$ & 0.01 & $0.020-0.085$ & 0.04 & - & - \\
\hline Pinonaldehyde & $0.06-0.67$ & 0.25 & $0.012-0.51$ & 0.04 & $0.46-2.5$ & 1.1 \\
\hline Nonanal & $0.16-0.82$ & 0.32 & $0.24-1.1$ & 0.6 & $0.03-0.37$ & 0.15 \\
\hline
\end{tabular}

${ }^{\mathrm{a}}$ Concentrations given in units of $\mathrm{ng} \mathrm{m}^{-3}$. Concentration ranges represent the 10 th and 90 th percentile. Data for acetaldehyde and nopinone at selected sites is absent owing to chromatographic interferences.

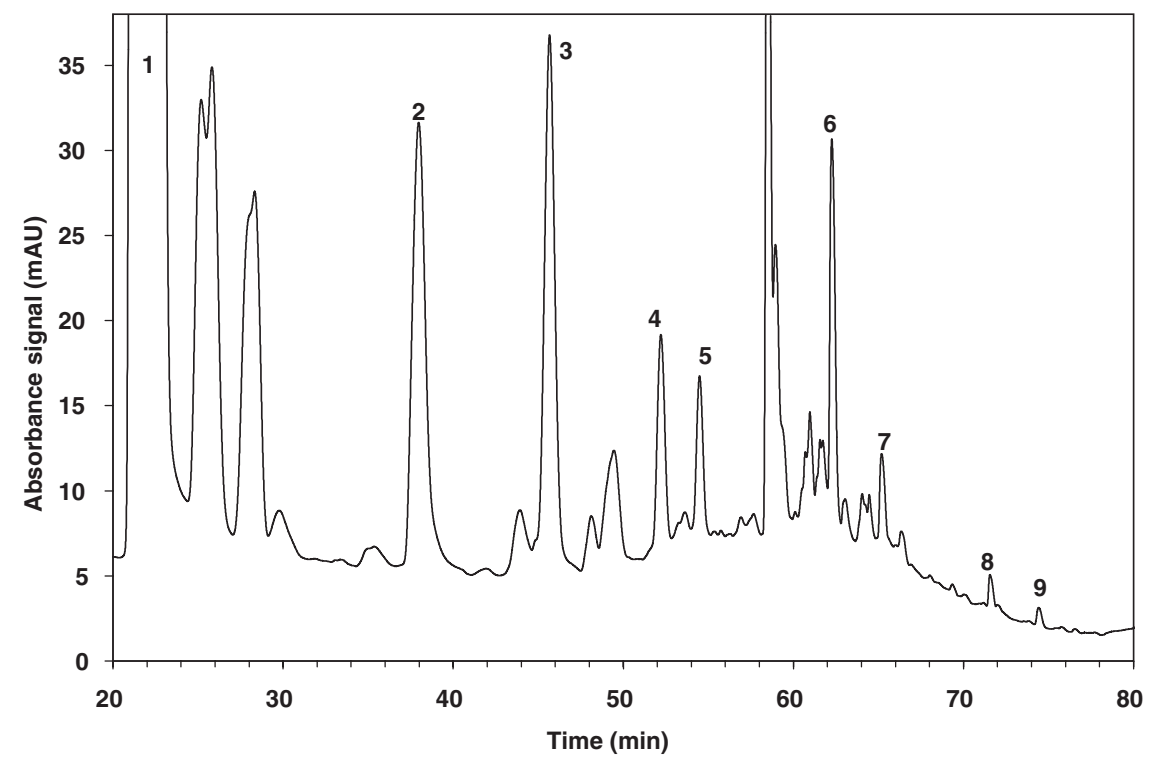

FIGURE 8 Sample chromatogram obtained during the Pacific 2001 field study. Peak labels: 1, 2,4-DNPH derivatizing agent; 2 . formaldehyde-DNPH; 3, acetaldehyde-DNPH; 4, acetone-DNPH; 5, propanal-DNPH; 6, glyoxal-DNPH; 7, methylglyoxal-DNPH; 8, pinonaldehyde-DNPH; 9, nonanl-DNPH.

several obvious trends are noted, and warrant further examination. Carbonyls such as formaldehyde, acetaldehyde, acetone, and propanal are the dominant species at all three sites, with median concentrations ranging from 1.1 to $33.9 \mathrm{ng} \mathrm{m}^{-3}$. These compounds can arise from a multitude of anthropogenic and biogenic sources, as well as secondary processes, which makes a source appointment for these compounds extremely difficult. Other species such as glyoxal and methylglyoxal have more distinct sources. These two dicarbonyls can arise from photo-oxidation of a number of anthropogenically emitted aromatic hydrocarbons [42-44]. Concentrations of these dicarbonyls in particulate matter significantly increase as the air mass travels up the valley from the urban Vancouver site $\left(0.78\right.$ and $0.1 \mathrm{ng} \mathrm{m}^{-3}$ median $)$ to the biogenic Sumas mountain site (1.7 and $0.47 \mathrm{ng} \mathrm{m}^{-3}$ median). This is, perhaps, an indication of 
secondary processes occurring along the valley, associated with emissions from the urban centre up wind. Pinonaldehyde and nopinone are known photo-oxidation products of $\alpha$ - and $\beta$-pinene [23,24], and have also been detected in particulates during the Pacific 2001 study. Levels of pinonaldehyde are significantly higher than nopinone levels at all sites, likely a result of a significantly higher $\alpha$-pinene concentration in the gas phase. Not surprisingly, concentrations of biogenic compounds, such as pinonaldehyde, are substantially higher at the Sumas site, which is heavily forested. Median values for pinonaldehyde at Sumas Mountain $\left(1.1 \mathrm{ng} \mathrm{m}^{-3}\right)$ are a factor of four times greater than those observed in Vancouver $\left(0.25 \mathrm{ng} \mathrm{m}^{-3}\right)$. In general, most species observed in the particulate-phase have their highest median values at the Sumas site, with the exception of nonanal. This may be indicative of a distinctly different formation and/or partitioning process occurring for this compound. Detailed analysis of the entire data set is ongoing.

\section{Artifacts and Filter Sampling}

It has been shown that when using the sampling techniques employed here, it may not be possible to disregard positive and negative artifacts [42-49] for various classes of compounds. However, it has also been shown that adsorption of organic vapors onto filter material resulting in a positive bias may be reduced by utilizing Tefloncoated filter materials $[47,49,50]$ over quartz filters, likely a result of Teflon's chemical inertness and smaller surface area. Other artifacts, which include volatilization of organic compounds from the particulate/filter material during sampling, and reactions of the particulate sample with gas-phase oxidants, may also result in further uncertainty. However, the total uncertainty with respect to sampling artifacts is not well defined, and no information is available regarding these processes, and their effect on the perceived particulate-phase carbonyl concentrations. Owing to the presence of artifacts, derived concentrations of particulate-phase carbonyls presented in this paper may be subject to the above uncertainties. Experiments are currently being conducted in an effort to quantify this effect, if any. However, other aspects of this method, including extraction, pre-concentration, detection, and reagent preparation can be applied to any sampling method, provided that the particulates are collected on a filter material. This may include sampling with combination denuders, filters and sorbents of varying geometry.

\section{Acknowledgments}

For funding, we acknowledge the National Science and Engineering Research Council of Canada and the Canadian Foundation for Climate and Atmospheric Sciences. We thank our partners in the Pacific 2001 study, the Meteorological Service of Canada and Greater Vancouver Regional District. We also acknowledge the assistance of Brian McCarry for collaborative filter cleaning protocols. Finally we acknowledge Filomena Chaves for significant editing of this article.

\section{References}

[1] S. Twomey, Atmos. Environ., 25A, 2435-2442 (1991).

[2] C. Pilinis, S. Pandis and J.H. Seinfeld, J. Geophys. Res., 100, 18739-18754 (1995). 
[3] J. Haywood and O. Boucher, Rev. Geophys., 38, 513-543 (2000).

[4] A. Eldering, S.M. Larson, J.R. Hall, K.J. Hussey and G.R. Cass, Environ. Sci. Technol., 27, 626-635 (1993).

[5] A. Eldering and G.R. Cass, J. Geophys. Res., 101, 19343-19369 (1996).

[6] C.S. Yuan, C.G. Lee, J.C. Chang, S.H. Liu, C. Yuan and H.Y. Yang, Proceedings of the 93rd Air and Waste Management Associations Annual Conference and Exhibition, 18-22 June 2000, Salt Lake City, Utah, paper \#87 on CD-ROM, Air \& Waste Management Association, Pittsburgh, PA (2000).

[7] J. Schwartz and D.W. Dockery, Am. J. Epidemiol., 135, 12-19 (1992).

[8] D.W. Dockery, C.A. Pope III, X. Xu, J.D. Spengler, J.H. Ware, M.E. Fay, B.J. Ferris and F.E. Speizer, N. Engl. J. Med., 39, 1753-1759 (1993).

[9] EPA, Air Quality Criteria for Particulate Matter, EPA/600/p-95/001CF. Environmental Protection Agency, Washington, DC (1996).

[10] F.M. Bowman, J.R. Odum, J.H. Seinfeld and S. Pandis, Atmos. Environ., 31, 3921-3931 (1997).

[11] J.F. Pankow, Atmos. Environ., 28, 189-193 (1994).

[12] J.F. Pankow, Atmos. Environ., 28, 185-188 (1994).

[13] J.F. Pankow, Atmos. Environ., 21, 2275-2283 (1987).

[14] M. Jang and R.M. Kamens, Environ. Sci. Technol., 35, 3626-3639 (2001).

[15] R.M. Kamens and M. Jaoui, Environ. Sci. Technol., 35, 1394-1405 (2001).

[16] M. Jang and R.M. Kamens, Environ. Sci. Technol., 35, 4758-4766 (2001).

[17] K. Kawamura, H. Kasukabe and L. Barrie, Atmos. Environ., 30, 1709-1722 (1996).

[18] K. Kawamura, Anal. Chem., 65, 3505-3511 (1993).

[19] J. Yu, R.J. Griffin, D.R. Cocker III, R.C. Flagan, J.H. Seinfeld and P. Blanchard, Geophys. Res. Let., 26, 1145-1148 (1999).

[20] I.G. Kavouras, N. Mihalopoulos and E.G. Stephanou, Geophys. Res. Let., 26, 55-58 (1999).

[21] H. Satsumabayashi, H. Kurita, Y. Yokouchi and H. Ueda, Atmos. Environ., 24A, 1443-1450 (1990).

[22] Y. Yokouchi and Y. Ambe, Atmos. Environ., 19, 1271-1276 (1985).

[23] J. Yu, D.R. Cocker III, R.J. Griffin, R.C. Flagan and J.H. Seinfeld, J. Atmos. Chem., 34, 207-258 (1999).

[24] B.R. Larsen, D. Di Bella, M. Glasius, R. Winterhalter, N.R. Jensen and J. Hjorth, J. Atmos. Chem., 38, 231-276 (2001).

[25] H.J. Forstner, R.C. Flagan and J.H. Seinfeld, Environ. Sci. Technol., 31, 1345-1358 (1997).

[26] E. Grosjean and D. Grosjean, Int. J. Environ. Anal. Chem., 61, 343-360 (1995).

[27] D.F. Smith, T. Kleindienst and E.E. Hudgens, J. Chromatogr., 483, 431-436 (1989).

[28] D. Grosjean, Environ. Sci. Technol., 24, 1428-1423 (1990).

[29] T.E. Kleindienst, P.B. Shepson, C.M. Nero, R.R Arnts, S.B. Tejada, G.I. Mackay, L.K. Mayne, H.I. Schiff and J.A. Lind, Atmos. Environ., 22, 1931-1939 (1988).

[30] S. Holliker, M. Oehme and C. Dye, Anal. Chem., 70, 1979-1985 (1998).

[31] Gy. Kiss, B. Varga, Z.V. Puchony, A. Gelencser, Z. Krivacsy and J. Hlavay, Talanta, 48, 755-762 (1999).

[32] J.B. De Andrade, H.L.C. Pinheiro and M.V. Andrade, Int. J. Environ. Anal. Chem., 52, 49-56 (1993).

[33] D. Grosjean, Eniviron. Sci. Technol., 16, 254-262 (1982).

[34] M. Glasius, A. Calogirou, N.R. Jensen, J. Hjorth and C.J. Nielsen, Int. J. Chem. Kinet., 29, 527-533 (1997).

[35] E. Grosjean and D. Grosjean, Int. J. Environ. Anal. Chem., 61, 47-64 (1995).

[36] R. Sander, Compilation of Henry's Law Constants for Inorganic And Organic Species of Potential Importance in Environmental Chemistry, Ver. 3. Max-Planck Institute for Chemistry, Mainz Germany (1999).

[37] I. Ogawa and J.S. Fritz, J. Chromatogr., 329, 81-89 (1985).

[38] K. Takami, K. Kuwata, A. Sugimae and M. Nakamoto, Anal. Chem., 57, 243-245 (1985).

[39] E. Cotsaris and B.C. Nicholson, Analyst, 118, 265-268 (1993).

[40] R.J. Kieber and K. Mopper, Environ. Sci. Technol., 24, 1477-1481 (1990).

[41] X. Zhou and K. Mopper, Environ. Sci. Technol., 24, 1482-1485 (1990).

[42] E.C. Tuazon, R. Atkinson, H. MacLeod, H.W. Biermann, A.M. Winer, W.P. Carter and J.N. Pitts Jr., Environ. Sci. Technol., 18, 981-984 (1984).

[43] H. Bandow and N. Washida, Bull. Chem. Soc. Jpn., 58, 2541-2548 (1985).

[44] H. Bandow and N. Washida, Bull. Chem. Soc. Jpn., 58, 2549-2555 (1985).

[45] R.E. Clement, F.W. Karasek, W.D. Bowers and M.L. Parsons, J. Chromatogr., 190, 136-140 (1980).

[46] D.J. Eatough, A. Wadsworth, D.A. Eatough, J.W. Crawford, L.D. Hansen and E.A. Lewis, Atmos. Environ., 27A, 1213-1219 (1993).

[47] B.T. Mader and J.F. Pankow, Environ. Sci. Technol., 35, 3422-3432 (2001).

[48] P. Masia, I. Allegrini, R.V. Gagliardi and R.E. Hillamo, J. Aerosol Sci., 28, S617-S618 (1997).

[49] S.R. McDow and J.J. Huntzicker, Atmos. Environ., 24A, 2563-2571 (1990).

[50] B.J. Turpin, J.J. Huntzicker and S.V. Hering, Atmos. Environ., 28, 3061-3071 (1994). 